ZAMM · Z. angew. Math. Mech. 77 (1997) 6, 467-475

Vrahatis, M. N.; Grapsa, T. N.; Ragos, O.; Zafiropoulos, F. A.

\title{
On the Localization and Computation of Zeros of Bessel Functions
}

The topological degree of a continuous mapping is implemented for the calculation of the total number of the simple real zeros within any interval of the Bessel functions of first and second kind and their derivatives. A new algorithm, based on this implementation, is given for the localization and isolation of these zeros. Furthermore, a second algorithm is presented for their computation employing a modified bisection method. The only information required for this computation is the algebraic signs of function values. Moreover, lower and upper bounds of a zero can also be obtained.

MSC (1991): 33C10, 65D20, 34B30

\section{Introduction}

The study of the Helmholtz equation in cylindrical coordinates eventually leads to the well-known Bessel equation

$$
x^{2} u^{\prime \prime}(x)+x u^{\prime}(x)+\left(x^{2}-v^{2}\right) u(x)=0,
$$

the solutions of which are

a) the Bessel function of order $v$ and argument $x$ of the first kind given by the series

$$
J_{\nu}(x)=\frac{x^{\nu}}{2^{\nu}} \sum_{m=0}^{\infty} \frac{(-1)^{m} x^{2 m}}{2^{2 m} m ! \Gamma(\nu+m+1)},
$$

which converges for every $x$ and

b) the Bessel function of order $v$ and argument $x$ of the second kind expressible in terms of functions of the first kind by the formula

$$
Y_{\nu}(x)=\left\{\begin{array}{cc}
\frac{J_{v}(x) \cos v \pi-J_{-\nu}(x)}{\sin v \pi}, & v \text { non-integral }, \\
\lim _{n \rightarrow v} \frac{J_{n}(x) \cos n \pi-J_{-n}(x)}{\sin n \pi}, & v=0,1,2, \ldots .
\end{array}\right.
$$

Regarding the zeros $j_{v}, j_{v}^{\prime}, y_{v}$, and $y_{v}^{\prime}$ of the functions $J_{v}(x), J_{v}^{\prime}(x), Y_{v}(x)$, and $Y_{v}^{\prime}(x)$, respectively, the following holds (cf., e.g., [5, 28]): For any real value of $\nu$ these functions have infinitely many real zeros all of which are simple, with the possible exception of $x=0$. Especially, for $v \geq-1, J_{\nu}(x)$ has only real zeros. If $v \geq 0$, all zeros of $J_{v}^{\prime}(x)$ are real.

For non-negative $v$, the $k$-th positive zeros of the above functions are denoted correspondingly by $j_{v, k}, j_{v, k}^{\prime}, y_{v, k}$, and $y_{v, k}^{\prime}$. They are interlaced according to the inequalities

$$
\begin{aligned}
& j_{v, 1}<j_{v+1,1}<j_{v, 2}<j_{v+1,2}<j_{v, 3}<\ldots \\
& y_{v, 1}<y_{v+1,1}<y_{v, 2}<y_{v+1,2}<y_{v, 3}<\ldots, \\
& v \leq j_{v, 1}^{\prime}<y_{v, 1}<y_{v, 1}^{\prime}<j_{v, 1}<j_{v, 2}^{\prime}<y_{v, 2}<y_{v, 2}^{\prime}<j_{v, 2}<j_{v, 3}^{\prime}<\ldots
\end{aligned}
$$

The mathematical problem of localizing and computing zeros of Bessel functions, encountered in many fields in science and engineering, has drawn a lot of attention and has evolved to a rather specialized branch of mathematics.

In the present paper we implement the concept of the topological degree to calculate the total number of real roots of Bessel functions within a predetermined interval and to isolate each one of them. For this purpose we use PICARD's extension [16, 17, 7, 8, 21] and either Kronecker theory or KEARFOTT's degree computation method. Once a zero is isolated, it can be computed numerically, utilizing a modified bisection method, to any accuracy (subject to relative machine precision). Thus, we propose two algorithms, one for the isolation and one for the computation of a real zero of a Bessel function. Upper and lower bounds for any root can also be produced.

\section{The topological degree for the localization of zeros}

Definition 1: Suppose that the function $F_{n}=\left(f_{1}, \ldots, f_{n}\right): \overline{\mathscr{D}}^{n} \subset \mathbf{R}^{n} \rightarrow \mathbb{R}^{n}$ is twice continuously differentiable in the domain $\mathscr{D}^{n}$ the boundary of which is denoted by $b\left(\mathscr{D}^{n}\right)$. Suppose further that the solutions of the equation

$$
F_{n}(x)=\mathcal{O}_{n}
$$


where $\mathcal{O}_{n}=(0, \ldots, 0)$ denotes the origin of $\mathbb{R}^{n}$, are not located on $b\left(\mathscr{D}^{n}\right)$, and they are simple, i.e., the Jacobian determinant of $F_{n}$ at these solutions is non-zero. Then the topological degree of $F_{n}$ at $\mathcal{O}_{n}$ relative to $\mathscr{D}^{n}$ is denoted by $\operatorname{deg}\left[F_{n}, \mathscr{D}^{n}, \mathcal{O}_{n}\right]$ and can be defined by the following sum:

$$
\operatorname{deg}\left[F_{n}, \mathscr{D}^{n}, \mathcal{O}_{n}\right]=\sum_{x \in F_{n}^{-1}\left(\mathcal{O}_{n}\right)} \operatorname{sgn} J_{F_{n}}(x)
$$

where $J_{F_{n}}$ indicates the determinant of the Jacobian matrix, and sgn defines the well known sign function with values

$$
\operatorname{sgn} \psi=\left\{\begin{array}{rll}
-1, & \text { if } & \psi<0 \\
0, & \text { if } & \psi=0 \\
1, & \text { if } & \psi>0
\end{array}\right.
$$

The above definition can be generalized when $F_{n}$ is only continuous [6, 14].

Kronecker's theorem $[2,6,14]$ states that equation (4) has at least one root in $\mathscr{D}^{n}$ if $\operatorname{deg}\left[F_{n}, \mathscr{D}^{n}, \mathcal{O}_{n}\right] \neq 0$.

The definition of the topological degree actually indicates that its value is equal to the number of simple solutions of equation (4) for which the Jacobian determinant is positive, minus the number of simple solutions for which the Jacobian is negative. Evidently, if all of them give the same Jacobian sign, then the total number $\mathscr{N}^{r}$ of simple roots of $F_{n}(x)$ can be obtained by the value of $\operatorname{deg}\left[F_{n}, \mathscr{D}^{n}, \mathscr{O}_{n}\right]$. To this end PICARD has considered the following extensions of the function $F_{n}$ and the domain $\mathscr{D}^{n}$ :

$$
F_{n+1}=\left(f_{1}, \ldots, f_{n}, f_{n+1}\right): \mathscr{D}^{n+1} \subset \mathbb{R}^{n+1} \rightarrow \mathbb{R}^{n+1},
$$

where $f_{n+1}=y J_{F_{n}}$ and $\mathscr{D}^{n+1}$ is the direct product of the domain $\mathscr{D}^{n}$ with an arbitrary interval of the real $y$-axis containing the point $y=0$. Then the following system of equations,

$$
\begin{aligned}
& f_{i}\left(x_{1}, x_{2}, \ldots, x_{n}\right)=0, \quad i=1, \ldots, n, \\
& y J_{F_{n}}\left(x_{1}, x_{2}, \ldots, x_{n}\right)=0,
\end{aligned}
$$

possesses the same simple roots with $F_{n}(x)$, provided $y=0$. Also, it is easily seen that the Jacobian of (7) is equal to $J_{F_{n}(x)}^{2}$ which is always positive. Thus we conclude that the total number $\mathscr{N}^{r}$ of solutions of equation (4) is

$$
\mathscr{N}^{r}=\operatorname{deg}\left[F_{n+1}, \mathscr{D}^{n+1}, \mathcal{O}_{n+1}\right] \text {. }
$$

\subsection{Kronecker integral approach}

The topological degree can be represented by the Kronecker integral as follows:

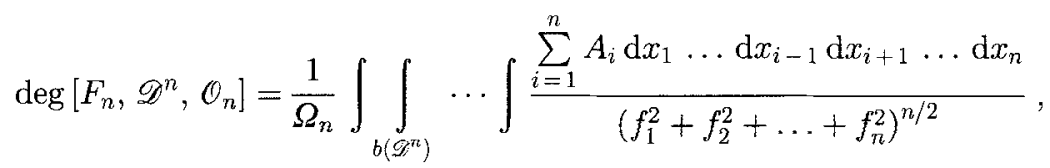

where $A_{i}$ define the following determinants:

$$
A_{i}=(-1)^{n(i-1)}\left|\begin{array}{ccccccc}
f_{1} & \frac{\partial f_{1}}{\partial x_{1}} & \ldots & \frac{\partial f_{1}}{\partial x_{i-1}} & \frac{\partial f_{1}}{\partial x_{i+1}} & \ldots & \frac{\partial f_{1}}{\partial x_{n}} \\
f_{2} & \frac{\partial f_{2}}{\partial x_{1}} & \ldots & \frac{\partial f_{2}}{\partial x_{i-1}} & \frac{\partial f_{2}}{\partial x_{i+1}} & \ldots & \frac{\partial f_{2}}{\partial x_{n}} \\
\vdots & \vdots & \ddots & \vdots & \vdots & \ddots & \vdots \\
f_{\eta z} & \frac{\partial f_{n}}{\partial x_{1}} & \cdots & \frac{\partial f_{n}}{\partial x_{i-1}} & \frac{\partial f_{n}}{\partial x_{i+1}} & \cdots & \frac{\partial f_{n}}{\partial x_{n}}
\end{array}\right|,
$$

and $\Omega_{n}$ denotes the surface of a hypersphere in $\mathbb{R}^{n}$ with radius one, i.e.: $\Omega_{n}=2 \pi^{n / 2} / \Gamma(n / 2)$.

The Kronecker integral has been numerically approximated with Gauss-Legendre quadrature [13].

In the present paper we study the zeros of a Bessel function, so we focus on the problem of calculating the total number of simple roots of a real function $f(x)$, defined in a predetermined interval $[a, b]$ and twice continuously differentiable in $(a, b)$, where $a$ and $b$ are arbitrarily chosen so that $f(a) f(b) \neq 0$.

According to Picard's extension we consider the function $F_{2}=\left(f_{1}, f_{2}\right): \mathscr{P}^{2} \subset \mathbb{R}^{2} \rightarrow \mathbb{R}^{2}$ and the corresponding system

$$
f_{1}(x, y)=f(x)=0, \quad f_{2}(x, y)=y f^{\prime}(x)=0,
$$

where the prime denotes differentiation, and $\mathscr{P}^{2}$ is the rectangular parallelepiped $[a, b] \times[-\xi, \xi]$ in the $(x, y)$-plane with $\xi$ an arbitrary positive constant. Since the roots are simple, which means $f^{\prime}(x) \neq 0$ for $x \in f^{-1}(0)$, it is easily seen that the solutions of system $(10)$ in $\mathscr{P}^{2}$, and those of $f(x)=0$ in $(a, b)$ are the same. Also, since $J_{F_{2}}=f^{\prime 2}$, the total 
number of simple zeros $\mathscr{N}^{r}$ of $f(x)$ in $(a, b)$ is given by

$$
\mathscr{N}^{r}=\operatorname{deg}\left[F_{2}, \mathscr{P}^{2}, \mathcal{O}_{2}\right] .
$$

For the computation of the topological degree of $F_{2}$ we apply Kronecker's integral (9) for $n=2$. Using the relations $\mathrm{d} f_{j}=\frac{\partial f_{j}}{\partial x_{1}} \mathrm{~d} x_{1}+\frac{\partial f_{j}}{\partial x_{2}} \mathrm{~d} x_{2}, j=1,2$, we obtain

$$
\mathscr{N}^{r}=\frac{1}{2 \pi} \oint_{b\left(\mathscr{P}^{2}\right)} \frac{f_{1} \mathrm{~d} f_{2}-f_{2} \mathrm{~d} f_{1}}{f_{1}^{2}+f_{2}^{2}}=\frac{1}{2 \pi} \oint_{b\left(\mathscr{P}^{2}\right)} \mathrm{d} \arctan \left(\frac{f_{2}}{f_{1}}\right) .
$$

Replacing $f_{1}$ and $f_{2}$ by virtue of (10) and performing the integration in (12) we finally get

$$
\mathscr{N}^{r}=-\frac{1}{\pi}\left[\xi \int_{a}^{b} \frac{f(x) f^{\prime \prime}(x)-f^{\prime 2}(x)}{f^{2}(x)+\xi^{2} f^{\prime 2}(x)} \mathrm{d} x-\arctan \left(\frac{\xi f^{\prime}(b)}{f(b)}\right)+\arctan \left(\frac{\xi f^{\prime}(a)}{f(a)}\right)\right] .
$$

Remark 1: It has been explicitly shown by PICARD [16, 17] that relation (13) is independent of the value of $\xi$.

The above developed method is applied in the sequel for the localization of the simple real zeros of the Bessel functions of the first kind, $J_{v}(x)$. In this case relation (13) becomes

$$
\mathscr{N}^{r}=-\frac{1}{\pi}\left[\xi \int_{a}^{b} G(x) \mathrm{d} x-\arctan \left(\frac{\xi J^{\prime} v(b)}{J_{\nu}(b)}\right)+\arctan \left(\frac{\xi J_{\nu}^{\prime}(a)}{J_{\nu}(a)}\right)\right],
$$

where the function $G(x)$, by means of equation (1), can be expressed in the following form:

$$
G(x)=\frac{\left(\frac{v^{2}}{x^{2}}-1\right) J_{v}^{2}(x)-\frac{1}{x} J_{v}(x) J_{v}^{\prime}(x)-J_{\nu}^{\prime 2}(x)}{J_{v}^{2}(x)+\xi^{2} J_{v}^{\prime 2}(x)} .
$$

\subsection{KeARFOTT's approach}

We could use any one of the degree computation methods (see e.g. $[22,13,10,11,23,24]$ ) to determine the total number of zeros by virtue of equation (11). Here we use KEARFOTT's method $[10,11,12]$ which compares favorably to other methods, in efficiency. This method is briefly described below.

Suppose that $S^{n-1}=\left\langle x_{1}, x_{2}, \ldots, x_{n}\right\rangle$ is an $(n-1)$-simplex $[22,10,11]$ in $\mathbb{R}^{n}$ and assume $F_{n}=$ $\left(f_{1}, f_{2}, \ldots, f_{n}\right): S^{n-1} \rightarrow \mathbb{R}^{n}$ is continuous. Then the range simplex associated with $S^{n-1}$ and $F_{n}$, denoted by $\mathscr{R}\left(S^{n-1}, F_{n}\right)$, is an $n \times n$ matrix with elements $\varrho_{i j}, 1 \leq i, j \leq n$, given by

$$
\varrho_{i j}=\left\{\begin{aligned}
1, & \text { if } \quad f_{j}\left(x_{i}\right) \geq 0 \\
-1, & \text { if } \quad f_{j}\left(x_{i}\right)<0
\end{aligned}\right.
$$

$\mathscr{R}\left(S^{n-1}, F_{n}\right)$ is called usable if one of the following conditions hold:

a) the elements $\varrho_{i j}$ of $\mathscr{R}\left(S^{n-1}, F_{n}\right)$, are:

$$
\varrho_{i j}=\left\{\begin{aligned}
1, & \text { if } \quad i \geq j \\
-1, & \text { if } \quad j=i+1
\end{aligned}\right.
$$

b) $\mathscr{R}\left(S^{n-1}, F_{n}\right)$ can be put into this form by a permutation of its rows.

When $\mathscr{R}\left(S^{n-1}, F_{n}\right)$ is usable, then the parity $\operatorname{Par}\left(\mathscr{R}\left(S^{n-1}, F_{n}\right)\right)$ is defined to be 1 , if the number of the permutations of the rows required to put $\mathscr{R}\left(S^{n-1}, F_{n}\right)$ into the form (17) is even. If this number is odd then $\operatorname{Par}\left(\mathscr{R}\left(S^{n-1}, F_{n}\right)\right)$ is defined to be -1 . For all other cases, we set $\operatorname{Par}\left(\mathscr{R}\left(S^{n-1}, F_{n}\right)\right)=0$. Suppose that $\mathscr{P}^{n}$ is an $n$-dimensional polyhedron for some $n \geq 2$, and that $\left\{S_{i}^{n-1}\right\}_{i=1}^{m}$ is a finite set of $(n-1)$-simplexes with disjoint interiors such that $\sum_{i=1}^{m} S_{i}^{n-1}=b\left(\mathscr{P}^{n}\right)$; then, under some assumptions regarding $S_{i}^{n-1}$, the value of the topological degree of $F_{n}$ at $\mathcal{O}_{n}$ relative to $\mathscr{P}^{n}$ can be obtained by the following relation:

$$
\operatorname{deg}\left[F_{n}, \mathscr{P}^{n}, \mathcal{O}_{n}\right]=\sum_{i=1}^{m} \operatorname{Par}\left(\mathscr{R}\left(S_{i}^{n-1}, F_{n}\right)\right) .
$$

Remark 2: Kearfott's degree computation method is very efficient and has the advantage that it requires only the signs of function values to be correct. 


\subsection{Isolating a zero}

With the above discussion in mind we give a description of our algorithm, in "pseudo-Pascal" (see e.g. [15]), independently of the degree computation method. In this algorithm $F_{\nu}(x)$ indicates the considered Bessel function of order $\nu$, and $(a, b)$ is a predetermined interval. It results in the total number $\mathcal{N}^{r}$ of roots which exist within $(a, b)$. Besides, it isolates one of them, bisecting the initial interval, and gives a lower bound $a_{k}$ and an upper one, $b_{k}$, for this root. If the isolation of the rest of them is required, the whole procedure has to be repeated successively for the remaining intervals $\left(a, a_{k}\right)$ and $\left(b_{k}, b\right)$.

Algorithm degree_isolate $\left(a, b, \mathscr{N}^{r}\right)$;

\{comment: This algorithm results in the total number $\mathcal{N}^{r}$ of roots of $F_{v}(x)$ in $(a, b)$ and isolates one of them\}.

procedure degree $\left(a_{k}, b_{k}, \mathscr{N}_{k}^{r}\right) ;\{$ comment: computes the total number of zeros $\}$

begin

Find $\mathcal{N}_{k}^{r}$, the number of zeros in $\left(a_{k}, b_{k}\right)$;

end $\{$ degree $\}$

procedure isolation $\left(a_{k}, b_{k}, \mathscr{N}_{k}^{r}\right) ;\left\{\right.$ comment: isolates a zero of $F_{v}(x)$ in $\left.\left(a_{k}, b_{k}\right)\right\}$

begin

while $\mathscr{N}_{k}^{r}>1$ do

begin

degree $\left(a_{k}, \frac{a_{k}+b_{k}}{2}, \mathscr{N}_{1}^{r}\right)$

$\mathscr{N}_{2}^{r}:=\mathscr{N}_{k}^{r}-\mathscr{N}_{1}^{r} ;$
if $\mathscr{N}_{2}^{r}=0$ then isolation $\left(a_{k}, \frac{a_{k}+b_{k}}{2}, \mathscr{N}_{1}^{r}\right) ;$

if $\mathscr{N}_{1}^{r}<\mathscr{N}_{2}^{r}$ and $\mathscr{N}_{1}^{r} \neq 0$ then isolation $\left(a_{k}, \frac{a_{k}+b_{k}}{2}, \mathscr{N}_{1}^{r}\right)$

else isolation $\left(\frac{a_{k}+b_{k}}{2}, b_{k}, \mathcal{N}_{2}^{r}\right)$;

end $\{$ while

end $\{$ isolation

begin \{degree_isolate $\}$

input $a, b$

degree $\left(a, b, \mathscr{N}^{r}\right)$

$\mathscr{N}_{*}^{r}:=\mathscr{N}^{r}$

if $\mathscr{N}^{r}>1$ then isolation $\left(a, b, \mathscr{N}^{r}\right)$;

output $a, b, \mathcal{N}_{*}^{r}$

end. $\{$ degree_isolate $\}$

\section{Computing roots of Bessel functions}

Having isolated one root of a Bessel function within an interval, we can use a modified version of the bisection method to compute it, as described in $[25,26]$. It is reported there that, in order to compute a solution of $f(x)=0$, where $f:[a, b] \subset \mathbb{R} \rightarrow \mathbb{R}$ is continuous, the following iterative formula can be used:

$$
x_{i+1}=x_{i}+c \operatorname{sgn} f\left(x_{i}\right) / 2^{i+1}, \quad i=0,1, \ldots,
$$

with $x_{0}=a$ and $c=\operatorname{sgn} f(a)(b-a)$. The iterations (19) converge to a root $r \in(a, b)$ if for some $x_{i}, i=1,2, \ldots$, there holds $\operatorname{sgn} f\left(x_{0}\right) \operatorname{sgn} f\left(x_{i}\right)=-1$.

The number of iterations, $\eta$, which are required in obtaining an approximate root $r^{*}$ such that $\left|r-r^{*}\right| \leq \varepsilon$ for some $\varepsilon \in(0,1)$, is given by

$$
\eta=\left\lceil\log _{2}\left((b-a) \varepsilon^{-1}\right)\right\rceil
$$

where the notation $[\cdot]$ refers to the smallest integer not less than the real number quoted.

It is evident from (19) that the only computable information required by the bisection method consists in the algebraic signs of the function $f$; so it can be applied to problems with imprecise function values. Moreover, the bisection method is a globally convergent method, it always converges within the given interval, and it is optimal [19, 20] in the sense that it possesses asymptotically the best rate of convergence. Also, it can be efficiently implemented for the computation of all the zeros and extrema of a function [9].

To apply our method, as previously mentioned, we only need the algebraic signs of the function values to be correct. The following results suggest the number of terms necessary in order to determine the algebraic signs of $J_{v}(x)$.

Proposition 1: The sign of the Bessel function $J_{v}$ for $v>-1$ is the same as the sign of the sum

$$
L_{\nu}(x)=\frac{x^{\nu}}{2^{\nu}} \sum_{m=0}^{M+K+1}(-1)^{m} a_{m}
$$


where $a_{m}$ are given by

$$
a_{m}=\frac{x^{2 m}}{2^{2 m} m ! \Gamma(\nu+m+1)},
$$

with

$$
M=\left\lceil\frac{-v-2+\sqrt{v^{2}+x^{2}}}{2}\right\rceil,
$$

and $K$ is defined so that the following relation holds:

$$
\left|a_{M+K+1}\right|<\left|\sum_{k=0}^{M+K}(-1)^{k} a_{k}\right| .
$$

Proof: We observe that

a) $a_{m}>0$

b) $\lim _{m \rightarrow \infty} a_{m}=0$,

c) $a_{m} \geq a_{m+1} \Leftrightarrow 4(m+1)(\nu+m+1)>x^{2}$.

Thus, the series

$$
R_{\nu}(x)=\sum_{m=M}^{\infty} \frac{(-1)^{m} x^{2 m}}{2^{2 m} m ! \Gamma(\nu+m+1)}
$$

is an alternating series which satisfies the three conditions of Leibniz's Theorem provided that

$$
m>\frac{-v-2+\sqrt{v^{2}+x^{2}}}{2} .
$$

The Alternating Series Estimation Theorem ensures that the absolute value of the $(M+K+1)$-th term is larger than that of the remainder of the series $R_{v}(x)$. Furthermore, the signs of the $(M+K+1)$-th term and of the remainder of the series $R_{\nu}(x)$ are the same. Now, by assumption (24) (which is fulfilled for some term $a_{i}$ since the sequence $\left\{a_{i}\right\}$ tends to zero when $i \rightarrow \infty)$, the sign of the Bessel function $J_{\nu}(x)$ is the same as the corresponding sign of $L_{v}(x)$. Thus the proposition is proved.

We can now give a description of our algorithm that computes, within a predetermined accuracy $\varepsilon$, a real root of the Bessel function $J_{v}(x)$, which has been isolated in the interval $\left(a_{k}, b_{k}\right)$.

Algorithm compute_zero $(a, b)$;

\{comment: This algorithm computes a zero of $J_{v}(x)$ in $(a, b)$. It uses $(19)$ and requires $\left.\Gamma(x), \varepsilon\right\}$.

procedure sign $(x, s) ;\left\{\right.$ comment: finds the sign $s$ of $\left.J_{v}(x)\right\}$

begin

$M:=\left\lceil\left(-v-2+\sqrt{v^{2}+x^{2}}\right) / 2\right\rceil ;$

$S:=0$;

for $m:=0$ to $M$ do $S:=S+(-1)^{m} x^{2 m+v} /\left(2^{2 m+v} m ! \Gamma(v+m+1)\right)$;

$m:=M+1$;

$T:=(-1)^{m} x^{2 m+v} /\left(2^{2 m+v} m ! \Gamma(v+m+1)\right) ;$

while $|T| \geq|S|$ do

begin

$$
\begin{aligned}
& S:=S+T \\
& m:=m+1 ; \\
& T:=(-1)^{m} x^{2 m+v} /\left(2^{2 m+v} m ! \Gamma(v+m+1)\right) ;
\end{aligned}
$$

end $\{$ while $\}$

Find $s:=\operatorname{sign}(S+T)$;

end $\{\operatorname{sign}\}$

begin $\{$ compute_zero $\}$

input $a_{k}, b_{k}, \varepsilon$

$x_{0}:=a_{k}$;

$\operatorname{sign}\left(x_{0}, s_{0}\right)$;

$c:=s_{0}\left(b_{k}-a_{k}\right)$;

for $i:=0$ to $\left\lceil\log _{2}\left(\left(b_{k}-a_{k}\right) \varepsilon^{-1}\right)\right\rceil$ do

begin

$\operatorname{sign}\left(x_{i}, s_{i}\right)$;

$x_{i+1}:=x_{i}+s_{i} c / 2^{i+1}$;

end

output $x_{i+1}$

end. $\{$ compute_zero $\}$ 
Remark 3: In order to obtain zeros of other Bessel functions, the above algorithm has to be adapted accordingly.

Evidently, utilizing the above results we are able to produce bounds for any real zero of a Bessel function. The following proposition proves it.

Proposition 2: Suppose that $f:[a, b] \subset \mathbb{R} \rightarrow \mathbb{R}$ is a continuous function and $(a, b)$ contains only one simple zero $x^{*}$ of $f$. Consider the sequence $(19)$ and its subsequences $\left\{x_{i}^{-}\right\},\left\{x_{i}^{+}\right\}$such that $\operatorname{sgn} f\left(x_{i}^{-}\right)=\operatorname{sgn} f(a)$, $\operatorname{sgn} f\left(x_{i}^{+}\right)=-\operatorname{sgn} f(a)=\operatorname{sgn} f(b)$. Then, $a_{\eta}$ and $b_{\eta}$, determined so that

$$
a_{\eta}=\max _{0 \leq i \leq \eta}\left\{x_{i}^{-}\right\}, \quad b_{\eta}=\min _{0 \leq i \leq \eta}\left\{x_{i}^{+}\right\},
$$

where $\eta$ is given by equation $(20)$, for any $\varepsilon \in(0,1)$, constitute a lower and an upper bound of the zero $x^{*}$ with $b_{\eta}-a_{\eta}<\varepsilon$.

Remark 4: The function $f$ can be replaced by any of the Bessel functions considered in this paper.

\section{Applications}

We have tested our algorithms with several random intervals $(a, b)$ and various Bessel functions. In each case we have calculated the total number $\mathcal{N}^{r}$ of roots of the respective Bessel function existing within $(a, b)$. For this calculation we have used both Kronecker's integral applied to Picard's extension and Kearfott's method. The computation of the integral of equation (14) depends on the integration method used. We have tried various integration methods. Here we exhibit the results obtained by Romberg's integration method. Also, we have observed that the speed of the numerical computation of the integral in equation (14) depends on the value of $\xi$. Our experience is that the fastest computation (using Romberg's method) can be obtained for $0.8 \leq \xi \leq 1.2$ while for other values the computational time increases (see Fig. 1). Kearfott's method is independent of the choice of $\xi$, but its speed depends on a stopping parameter $p$ and the maximum tree depth MD [11]. In our case, we have obtained accurate results even for small values of $p$ and $\mathrm{MD}$, as for instance $p=1, \mathrm{MD}=2$.

Table 1 presents the number of zeros of Bessel functions of various orders $v$ existing within some given intervals $(a, b)$, as well as the respective subintervals $\left(a_{k}, b_{k}\right)$, where exactly one root, $r_{k}$, exists.

In Table 2 we give the first ten zeros of several Bessel functions, chosen at random, computed by Algorithm compute_zero.

\begin{tabular}{|c|c|c|c|c|c|c|}
\hline & $a$ & $b$ & $\mathscr{N}^{r}$ & $a_{k}$ & $b_{k}$ & $r_{k}$ \\
\hline$J_{0}$ & 1 & 100 & 32 & 1.0000 & 4.09375 & 2.404825557695773 \\
\hline$J_{0.3}$ & 1 & 100 & 31 & 81.4375 & 87.62500 & 84.509788949453324 \\
\hline$J_{2.5}$ & 1 & 100 & 30 & 1.0000 & 7.18750 & 5.763459196894550 \\
\hline$J_{10}$ & 10 & 100 & 27 & 10.0000 & 15.62500 & 14.475500686554542 \\
\hline$J_{13.3}$ & 10 & 100 & 25 & 10.0000 & 21.25000 & 18.131465204981082 \\
\hline$J_{50}$ & 50 & 100 & 11 & 50.0000 & 62.50000 & 57.116899160119190 \\
\hline$J_{0}^{\prime}$ & 1 & 100 & 31 & 13.3750 & 19.56250 & 16.500922441528084 \\
\hline$J_{0.3}^{\prime}$ & 1 & 100 & 32 & 1.0000 & 4.09375 & 1.308699363719847 \\
\hline$J_{2.5}^{\prime .0}$ & 1 & 100 & 31 & 1.0000 & 7.18750 & 3.959527916501095 \\
\hline$J_{10}^{I .}$ & 10 & 100 & 27 & 10.0000 & 15.62500 & 12.128927704415439 \\
\hline$J_{13.3}^{\prime}$ & 10 & 100 & 26 & 10.0000 & 15.62500 & 15.617873137336602 \\
\hline$J_{50}^{\prime}$ & 50 & 100 & 11 & 50.0000 & 56.25000 & 53.502858820400364 \\
\hline$Y_{0}$ & 1 & 100 & 31 & 13.3750 & 19.56250 & 16.470630050877633 \\
\hline$Y_{0.3}$ & 1 & 100 & 31 & 1.0000 & 7.18750 & 4.301991992307308 \\
\hline$Y_{2.5}$ & 1 & 100 & 31 & 1.0000 & 7.18750 & 3.632797319831763 \\
\hline$Y_{10}$ & 10 & 100 & 27 & 10.0000 & 15.62500 & 11.770876674955581 \\
\hline$Y_{13.3}$ & 10 & 100 & 26 & 10.0000 & 15.62500 & 15.242738650014224 \\
\hline$Y_{50}$ & 50 & 100 & 11 & 50.0000 & 56.25000 & 52.997640387316651 \\
\hline$Y_{0}^{\prime}$ & 1 & 100 & 32 & 1.0000 & 4.09375 & 2.197141326031016 \\
\hline$Y_{0.3}^{\prime}$ & 1 & 100 & 31 & 81.4375 & 87.62500 & 84.503872394936382 \\
\hline$Y_{25}^{\prime}$ & 1 & 100 & 30 & 1.0000 & 7.18750 & 5.634296563929559 \\
\hline$Y_{10}^{\prime}$ & 10 & 100 & 27 & 10.0000 & 15.62500 & 14.353013743699869 \\
\hline$Y_{13.3}^{\prime \prime}$ & 10 & 100 & 25 & 10.0000 & 21.25000 & 18.006479815952494 \\
\hline$Y_{50}^{\prime \prime}$ & 50 & 100 & 11 & 50.0000 & 62.50000 & 56.962904275167517 \\
\hline
\end{tabular}




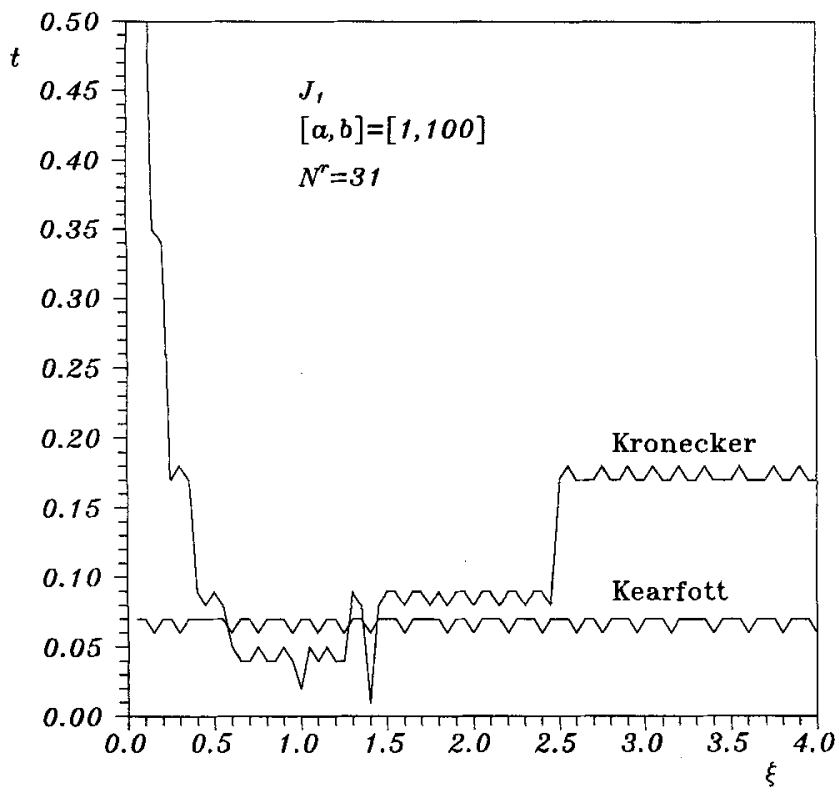

Fig. 1. Time required for the computation of the topological degree using Kronecker's integral versus $\xi$ and comparison with Kearfott's method

Table 2. Zeros of various Bessel functions computed by Algorithm compute_zero

\begin{tabular}{|c|c|c|c|c|c|}
\hline \multirow[t]{10}{*}{$J_{0}$} & 2.404825557695773 & \multirow[t]{10}{*}{$J_{2.5}$} & 5.763459196894550 & \multirow[t]{10}{*}{$J_{50}$} & 57.116899160119190 \\
\hline & 5.520078110286310 & & 9.095011330476354 & & 62.807698764835380 \\
\hline & 8.653727912911011 & & 12.322940970566584 & & 67.697408410764783 \\
\hline & 11.791534439014282 & & 15.514603010886749 & & 72.190366544011145 \\
\hline & 14.930917708487784 & & 18.689036355362817 & & 76.437072182667940 \\
\hline & 18.071063967910918 & & 21.853874222709777 & & 80.513239317465752 \\
\hline & 21.211636629879254 & & 25.012803202289602 & & 84.463252949061289 \\
\hline & 24.352471530749303 & & 28.167829707993626 & & 88.315711749199524 \\
\hline & 27.493479132040244 & & 31.320141707447189 & & 92.090274456391647 \\
\hline & 30.634606468431967 & & 34.470488331285007 & & 95.801108265953272 \\
\hline \multirow[t]{10}{*}{$J_{0}^{\prime}$} & 3.957678419314857 & \multirow{10}{*}{$J_{2.5}^{\prime}$} & 3.959527916501094 & \multirow[t]{10}{*}{$J_{50}^{\prime}$} & 53.502858820400364 \\
\hline & 7.086051060301773 & & 7.451610064214504 & & 60.112444427740558 \\
\hline & 10.222345043496417 & & 10.715647375791513 & & 65.317141149297498 \\
\hline & 13.361097473872762 & & 13.921686012308782 & & 69.981432989702090 \\
\hline & 16.500922441528084 & & 17.103359117208743 & & 74.338747166755810 \\
\hline & 19.641309700887942 & & 20.272369140216535 & & 78.493210918315127 \\
\hline & 22.782028047291558 & & 23.433926142067801 & & 82.501961830679640 \\
\hline & 25.922957653180932 & & 26.590716631086269 & & 86.400289204160094 \\
\hline & 29.064030252728390 & & 29.744270680556565 & & 90.211743342930555 \\
\hline & 32.205204116493269 & & 32.895525188224304 & & 93.952927913983330 \\
\hline \multirow[t]{10}{*}{$Y_{0}$} & 3.831705970207513 & \multirow[t]{10}{*}{$Y_{2.5}$} & 3.632797319831762 & \multirow[t]{10}{*}{$Y_{50}$} & 52.997640387316651 \\
\hline & 7.015586669815619 & & 7.367008971566918 & & 60.026319332799442 \\
\hline & 10.173468135062721 & & 10.663561390481999 & & 65.272723327026940 \\
\hline & 13.323691936314222 & & 13.883369775209742 & & 69.951692734372273 \\
\hline & 16.470630050877633 & & 17.072848832681667 & & 74.316349977805003 \\
\hline & 19.615858510468247 & & 20.246944819393926 & & 78.475187454486474 \\
\hline & 22.760084380592769 & & 23.412099866421985 & & 82.486831848982885 \\
\hline & 25.903672087618386 & & 26.571579024683013 & & 86.387212778756663 \\
\hline & 29.046828534916836 & & 29.727222608526206 & & 90.200199617262318 \\
\hline & 32.189679910974398 & & 32.880149822147309 & & 93.942572252611562 \\
\hline \multirow[t]{10}{*}{$Y_{0}^{\prime}$} & 2.197141326031016 & \multirow[t]{10}{*}{$Y_{2.5}^{\prime}$} & 5.634296563929560 & \multirow[t]{10}{*}{$Y_{50}^{\prime}$} & 56.962904275167517 \\
\hline & 5.429681040794136 & & 9.030901729624809 & & 62.748881669459305 \\
\hline & 8.596005868331170 & & 12.278862551656813 & & 67.661781418302983 \\
\hline & 11.749154830839879 & & 15.480654965172806 & & 72.164827720771193 \\
\hline & 14.897442128336724 & & 18.661308999166464 & & 76.417109157783180 \\
\hline & 18.043402276727858 & & 21.830389858741036 & & 80.496796665401667 \\
\hline & 21.188068934142211 & & 24.992411488536626 & & 84.449230155149340 \\
\hline & 24.331942571356901 & & 28.149798292577922 & & 88.303453538378357 \\
\hline & 27.475294980449235 & & 31.303973776577955 & & 92.079360103387758 \\
\hline & 30.618286491641110 & & 34.455830546321302 & & 95.791251970104038 \\
\hline
\end{tabular}




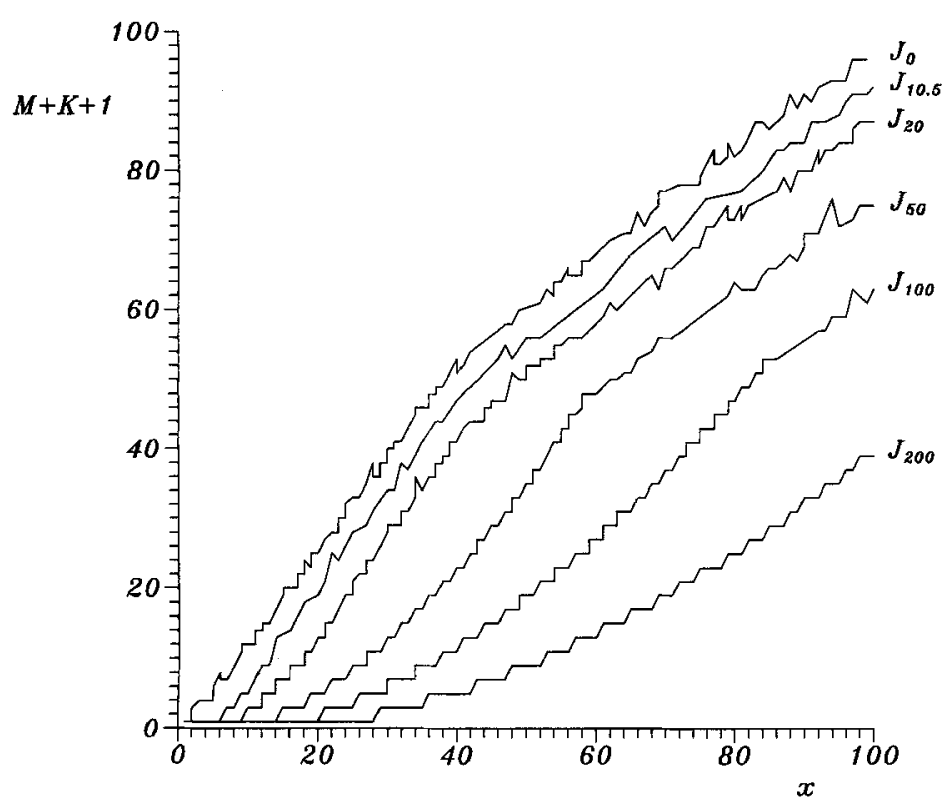

Fig. 2. The number of terms to be added for the determination of the sign of $J_{v}(x)$ versus $x$ for $v=0,10.5,20,50,100,200$

By virtue of Proposition 1, we have obtained the number of terms of $J_{v}$ required to obtain the corresponding algebraic sign for various values of the order $v$ and the argument $x$. As it is shown in Fig. 2, the number of terms decreases as the order increases.

\section{Concluding remarks}

An efficient method for locating, isolating, and computing real zeros of Bessel functions is described in this paper. It has been implemented and tested, and our experience is that it behaves predictably and accurately.

The first phase of this method (algorithm degree_isolate) exploits topological degree theory and especially Picard's extension to calculate the total number of real roots of Bessel functions within a predetermined interval and to isolate one of them. This procedure can be repeated for the isolation of each one of the zeros in this interval.

Once a zero is isolated, the second phase (algorithm compute-zero) is applied for its computation to any accuracy (subject to relative machine precision). This algorithm utilizes a modified bisection method. The only computable information required consists in the algebraic signs of the function and, consequently, it is not affected by imprecise function values. Moreover, it always converges rapidly to a zero within the initially specified region independently of the starting guess. It is also a globally convergent method, it can be applied to nondifferentiable continuous functions and does not involve derivatives or approximations of such derivatives. Furthermore, the number of iterations needed to compute a zero to a predetermined accuracy is a priori known.

The rootfinding portion of our method requires the smallest amount of function value information which is its algebraic sign. Using this and the stopping criterion (20), it computes a zero of a Bessel function within a given accuracy.

We have been able to calculate the total number of zeros by computing the value of the topological degree of (11) utilizing efficient methods such as STEnGER's [22], STYNES' [23, 24], or KEARFOTT's method [10, 11] (see also [1]), which are based only on sign calculations.

For large argument $x$, one can use the usual asymptotic expressions for Bessel functions [28]. Moreover, if one would like to compute zeros of a combination of Bessel functions which may not be an alternating series, the van Wijngaarden transformation can be used to convert this new series into an alternating one [18]. Furthermore, very accurate function values for the Bessel functions can be obtained by employing STEED's and TEMME's methods [18], or, alternatively, by means of Coulomb functions, employing BARNETT's procedure [3, 4].

When just one root is required, the isolation portion of our method can be avoided if the function values at the endpoints of the given interval are opposite (see [27] for extensions). This is so because, in this case, the modified bisection method always converges to a zero.

Since the first derivatives of Bessel functions are available, it is at the user's disposal to apply any other rootfinding method, as for instance Newton's method, to accelerate the convergence within the predetermined interval. In such a case, though, unless the starting point is close enough to a zero and certainly away from an extremum of the function, convergence is not ensured. Thus, a few initial steps of our method should be performed so that these conditions are satisfied.

At last, for any given interval $\left(a_{k}, b_{k}\right)$ containing a single zero of a Bessel function, Proposition 2 can provide a lower and an upper bound for this zero, theoretically as close to it as one desires, but practically as close to it as the accuracy of the calculations permits. 
Acknowledgements: We are indebted to Professor RALPH BAKER KEARFotT for kindly providing his Fortran version of a program described in [10].

\section{References}

1 ABerth, O.: Computation of topological degree using interval arithmetic, and applications. Math. Comput. 62 (1994), $171-178$.

2 Alexandroff, P.; Hopf, H.: Topologie. Springer-Verlag, Berlin 1935; reprinted: Bronx, Chelsea, New York 1965.

3 Barnett, A. R.: KLEIN : Coulomb functions for real $\lambda$ and positive energy to high accuracy. Comput. Phys. Commun. 24 (1981), 141-159.

4 BARnEtT, A. R.: COULFG: Coulomb and Bessel functions and their derivatives, for real arguments, by Steed's method. Comput. Phys. Commun. 27 (1982), 147-166.

5 Courant, R.; Hrlbert, D.: Methods of mathematical physics. Vol. 1. Wiley-Interscience, New York 1953.

6 CRonin, J.: Fixed points and topological degree in nonlinear analysis. Mathematical Surveys No. 11, Amer. Math. Soc., Providence, Rhode Island 1964.

7 Hoenders, B. J.; Slump, C. H.: On the calculation of the exact number of zeroes of a set of equations. Computing 30 (1983), $137-147$.

8 HoEnders, B. J.; SLUMP, C. H.: On the determination of the number and multiplicity of zeros of a function. Computing $\mathbf{4 7}$ (1992), 323-336.

9 Kavvadias, D.; VRahatis, M. N.: Locating and computing all the simple roots and extrema of a function. SIAM J. Sci. Comput. 17 (1996), 1232-1248.

10 Kearfott, R. B.: Computing the degree of maps and a generalized method of bisection. Ph. D. dissertation, Department of Mathematics, University of Utah, S.L.C. 1977.

11 Kearfott, R. B.: An efficient degree-computation method for a generalized method of bisection. Numer. Math. 32 (1979), $109-127$.

12 KEARFOTT, R. B.: private communication.

13 O'Neil, T.; Thomas, J.: The calculation of the topological degree by quadrature. SIAM J. Numer. Anal. 12 (1975), 673-680.

14 Ortega, J. M.; Rheinbolt, W. C.: Iterative solution of nonlinear equations in several variables. Academic Press, New York 1970.

15 Papadimitriou, C. H.; Steiglitz, K.: Combinatorial optimization: algorithms and complexity. Prentice-Hall, Inc., Englewood Cliffs, New Jersey 1982.

16 PiCARD, E.: Sur le nombre des racines communes à plusieurs équations simultanées. J. de Math. Pure et Appl., $4^{\mathrm{e}}$ série 8 (1892), $5-24$.

17 PICARD, E.: Traité d'analyse. 3rd ed., chap. 4.7, Gauthier-Villars, Paris 1922.

18 Press, W. H.; Teukolsky, S. A.; Vetterling, W. T.; Flannery, W. T.: Numerical recipes, the art of scientific computing. 2nd ed. Cambridge University Press, New York 1992.

19 Sikorski, K.: Bisection is optimal. Numer. Math. 40 (1982), 111-117.

20 Sikorski, K.; Trojan, G. M.: Asymptotic near optimality of the bisection method. Numer. Math. 57 (1990), $421-433$.

21 Slump, C. H.; Hoenders, B. J.: The determination of the location of the global maximum of a function in the presence of several local extrema. IEEE Trans. Inform. Theory IT-31 (1985), 490-497.

22 STEnger, F.: Computing the topological degree of a mapping in $\mathbb{R}^{n}$. Numer. Math. 25 (1975), 23-38.

23 STYNeS, M.: An algorithm for the numerical calculation of the degree of a mapping. Ph. D. dissertation, Department of Mathematics, Oregon State University, Corvallis 1977.

24 STynes, M.: An algorithm for numerical calculation of topological degree. Appl. Anal. 9 (1979), 63-77.

25 VRahatis, M. N.: Solving systems of nonlinear equations using the nonzero value of the topological degree. ACM Trans. Math. Software 14 (1988), 312-329.

26 VRAhATIS, M. N.: CHABIS: A mathematical software package for locating and evaluating roots of systems of nonlinear equations. ACM Trans. Math. Software 14 (1988), 330-336.

27 Vrahatis, M. N.: A short proof and a generalization of Miranda's existence theorem. Proc. Amer. Math. Soc. 107 (1989), $701-703$.

28 Watson, G. N.: A treatise on the theory of Bessel functions. Cambridge University Press, Cambridge 1966.

Received July 24, 1995 at ZAA; received at ZAMM September 1, 1995, accepted May 7, 1996

Address: Prof. Dr. M. N. Vrahatis, Dr. T. N. Grapsa, Dr. O. Ragos, Dr. F. A. Zafiropoulos, Department of Mathematics, University of Patras, GR-26110 Patras, Greece 